\title{
Application of harmonic balance method for solving non-linear equations of motion
}

\author{
Robert Kostek ${ }^{l, *}$ \\ ${ }^{1}$ University of Science and Technology, Al. Prof. S. Kaliskiego 7, 85-796 Bydgoszcz, Poland
}

\begin{abstract}
This article presents the advantages and limitations of a harmonic balance method applied for solving non-linear equations of monition. This method provides an opportunity to find stable and unstable periodic solutions, which was demonstrated for a few equations. An error of solution decreases rapidly with increase of number of harmonics for smooth time history of acceleration, which shows convergence; whereas, for discontinuous time histories, this method is not effective.
\end{abstract}

\section{Introduction}

Non-linear equations of motion are usually solved with implicit and explicit numerical methods, which integrate time histories. Typically the Runge-Kutta, polynomial or direct methods are used to solve the equations [1]. These numerical methods provide an opportunity to simulate chaotic and transient vibration; whereas stable periodic vibrations, are obtained after certain time, when transient component decreases to negligible value. Thus there appears to be some problems with simulation undamped and unstable vibrations, because it is known that numerical methods change the energy of simulated system, thus sometimes vibration amplitude rises and transient component remains large. These phenomena are coupled with stability of numerical methods [2]. Undamped vibrations can be simulated with numerical methods, which integrate time histories; nevertheless, additional procedures and skills are required. Whereas the Harmonic Balance Method (HBM) can be used to find stable and unstable cycles and their spectrums without these problems [3].

\section{Convergence of HBM}

The Harmonic Balance Method employs a Fourier series to estimate time histories of displacement, velocity and acceleration. These series are presented below:

$$
\begin{gathered}
x_{\mathrm{h}}=A_{0}+\sum A_{\mathrm{n}} \sin \left(\omega_{\mathrm{n}} \mathrm{t}+\phi_{\mathrm{n}}\right), \\
x_{\mathrm{h}}{ }^{\prime}=\Sigma A_{\mathrm{n}} \omega_{\mathrm{n}} \sin \left(\omega_{\mathrm{n}} \mathrm{t}+\phi_{\mathrm{n}}+\pi / 2\right), \\
x_{\mathrm{h}}{ }^{\prime}=\Sigma A_{\mathrm{n}} \omega_{\mathrm{n}}{ }^{2} \sin \left(\omega_{\mathrm{n}} \mathrm{t}+\phi_{\mathrm{n}}+\pi\right),
\end{gathered}
$$

where $x_{\mathrm{h}}$ - is displacement $\mathrm{m}, x_{\mathrm{h}}{ }^{\prime}$ - velocity $\mathrm{m} / \mathrm{s}, x_{\mathrm{h}}{ }^{\prime}$ - acceleration $\mathrm{m} / \mathrm{s}^{2}, A_{\mathrm{n}}$ - amplitude of $\mathrm{n}^{\text {th }}$ harmonic $\mathrm{m}, \omega_{\mathrm{n}}=\omega_{1} n-$ frequency of $\mathrm{n}^{\text {th }}$ harmonic $\mathrm{rad} / \mathrm{s}, \phi_{\mathrm{n}}-$ phase angle of $\mathrm{n}^{\text {th }}$ harmonic $\mathrm{rad}$, and $t$ - time in $\mathrm{s}$. These series are substituted in to the following equation of motion:

$$
x^{\prime \prime}=f\left(x, x^{\prime}, t\right) / m,
$$

where $m$ - is mass $\mathrm{kg}$. Acceleration can be computed both from equation (3) and (4), thus an error can be calculated from the following expression:

$$
E_{x}{ }^{\prime \prime(t)}=x_{\mathrm{h}}{ }^{\prime}-f\left(x_{\mathrm{h}}, x_{\mathrm{h}}, t\right) / m
$$

where $E_{\mathrm{x}}{ }^{\prime \prime}(t)$ - is an error. The objective function is the root mean square of this error $\operatorname{RMS}\left(E_{\mathrm{x}}{ }^{\prime}(t)\right)$. This leads to optimisation problem, where amplitudes $A_{0}, \ldots, A_{\mathrm{i}}$, phase angles $\phi_{1}, \ldots, \phi_{1}$, and frequencies $\omega_{1}, . ., \omega_{1}$ are variables. Summarising, HBM replace integration by optimisation.

\footnotetext{
${ }^{*}$ Corresponding author: robertkostek@o2.pl
} 
Convergence of harmonic balance method should be studied for non-linear equations, which have exact solutions. First, the equation with cubic non-linearity is studied:

$$
x,=\left(-k_{3} x^{3}\right) / m
$$

where $k_{3}$ - is stiffness co-efficient $\mathrm{N} / \mathrm{m}^{3}$. For three harmonics obtained solutions are near a numerical solution, which demonstrates convergence (Fig. 1). Time histories of $x_{\mathrm{h}}$ "' and $f\left(x_{\mathrm{h}}{ }^{\prime}, x_{\mathrm{h}}, t\right)$ are very similar, thus value of $\operatorname{RMS}\left(E_{\mathrm{x}},(t)\right)$ is small; nevertheless, some error of period is observed. It should be mentioned that, in spite of fact that the studied equation (6) is strongly non-linear, the amplitude of the first harmonic is the largest, whereas further amplitudes are small.
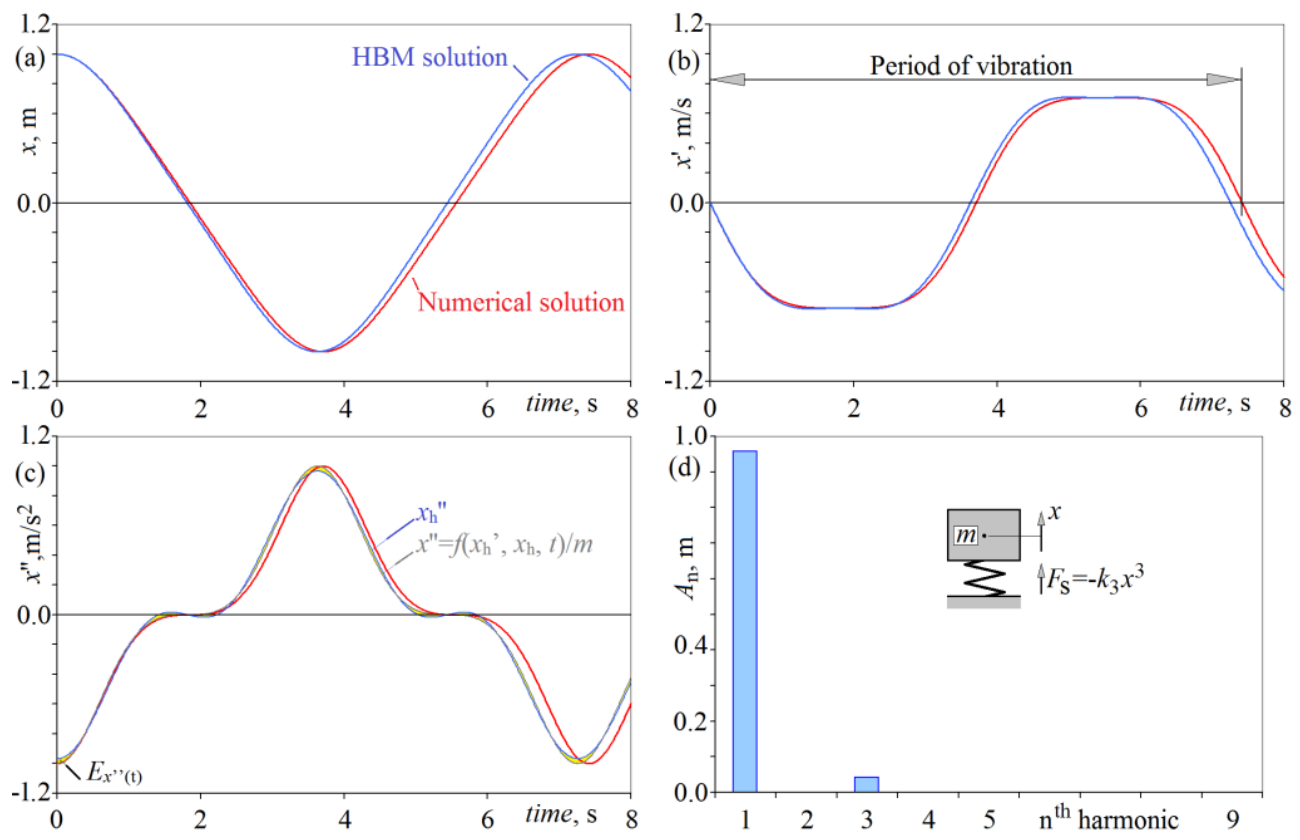

Fig. 1. Time histories of displacement (a), velocity (b), acceleration (c), and spectrum (d) obtained with HBM for the following conditions $m=1 \mathrm{~kg}, k_{3}=1 \mathrm{~N} / \mathrm{m}^{3}, x_{(\mathrm{t}=0)}=1 \mathrm{~m}$ and $x^{\prime}{ }_{(\mathrm{t}=0)}=0 \mathrm{~m} / \mathrm{s}$, Eq. (6).

Further, two errors can be studied: the error of period $E_{\mathrm{T}}$ and objective function $\operatorname{RMS}\left(E_{x},(\mathrm{t})\right) \cdot \operatorname{RMS}\left(E_{x},(\mathrm{t})\right)$ drops rapidly with an increase in the number of harmonic (Fig. 2a), thus for $n=7$ time histories are very similar to exact solution. The same situation is observed for error of period (Fig. 2b), which is expressed by the following equation:

$$
E_{\mathrm{T}}=\left|\left(T_{\mathrm{HBM}}-T\right) / T\right|,
$$

where $E_{\mathrm{T}}$ - denotes error of period, $T$ - is exact period and $T_{\mathrm{HBM}}$ - represents period obtained with HBM. The same phenomenon is observed for perturbation solution, where the error of period decreases with the increasing order of any solution. The HBM is effective in this case, because time histories are smooth and the amplitudes of higher harmonics are small. 

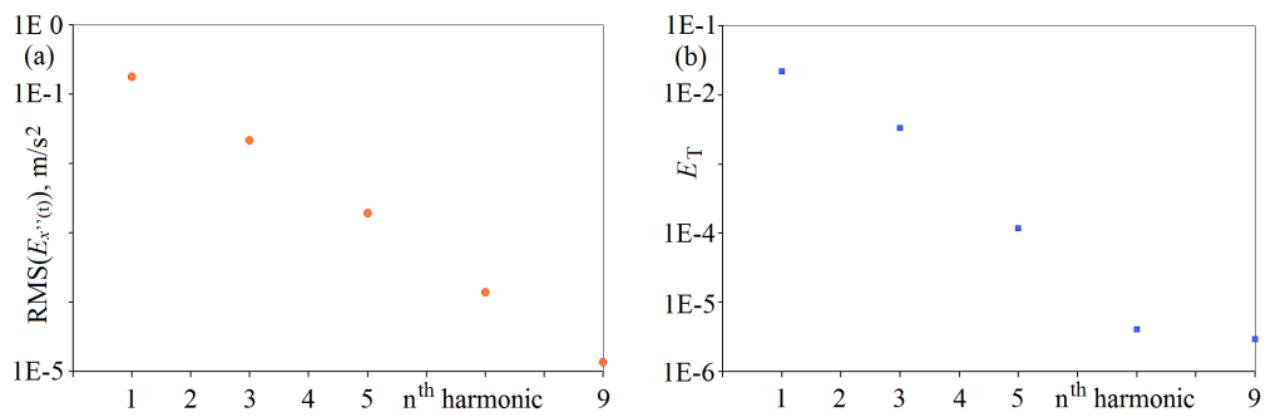

Fig. 2. $\operatorname{RMS}\left(E_{x^{\prime}}{ }^{\prime}(\mathrm{t})\right)(\mathrm{a})$ and error of period $E_{\mathrm{T}}(\mathrm{b})$ against number of harmonic.

Next vibrations described by the following equation are studied:

$$
x^{\prime \prime}=\left(-F_{0} \operatorname{sign}(x)\right) / \mathrm{m},
$$

where $F_{0}$ - denotes magnitude of potential force. This force is described by discontinuous function, thus the time history of acceleration is discontinuous, which introduces problems with convergence. The presented solution contains nine harmonics, thus it should be near exact and a numerical solution (Fig.3). For time histories of displacement, good agreement is observed, whereas for time histories of velocity, small differences are noticed. On the other hand differences between time histories of acceleration are obvious. Additional waves are observed, and jumps of acceleration are not well reflected. This shows a slow convergence of the Taylor series to discontinuous functions. Large number of harmonics should be used in this case, which is not effective. The same phenomenon is observed for Coulomb friction. Thus, another solution is applicable - the Walsh function. The spectrum of displacement is depicted in Fig.3d. The amplitude of the first harmonic is the largest, but higher harmonics play a significant role for time history of acceleration.

Next, two errors are studied - RMS $\left(E_{x}{ }^{\prime \prime}(\mathrm{t})\right)$ and $E_{\mathrm{T}}$. $\operatorname{RMS}\left(E_{x^{\prime \prime}(\mathrm{t})}\right)$ which drop very slowly with an increase in the number of harmonics (Fig. 4a), thus for $n=9$ differences between time histories of acceleration are observed. Whereas, $E_{\mathrm{T}}$ drops faster (Fig. $4 \mathrm{~b}$ ), and for nine harmonics period of vibration obtained with HBM is similar to exact period (Fig. 3c).

Summarising this section, HBM is effective for smooth time histories of acceleration, and periodic vibrations. The test problems were without damping force, thus were between stable and unstable regions. Undamped vibrations are difficult to simulation with methods, which integrate time histories.
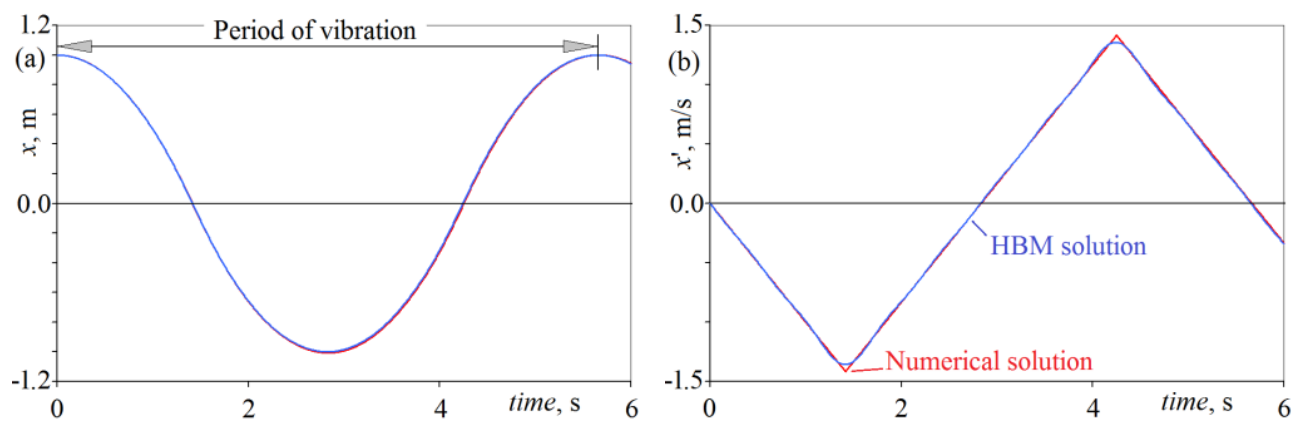

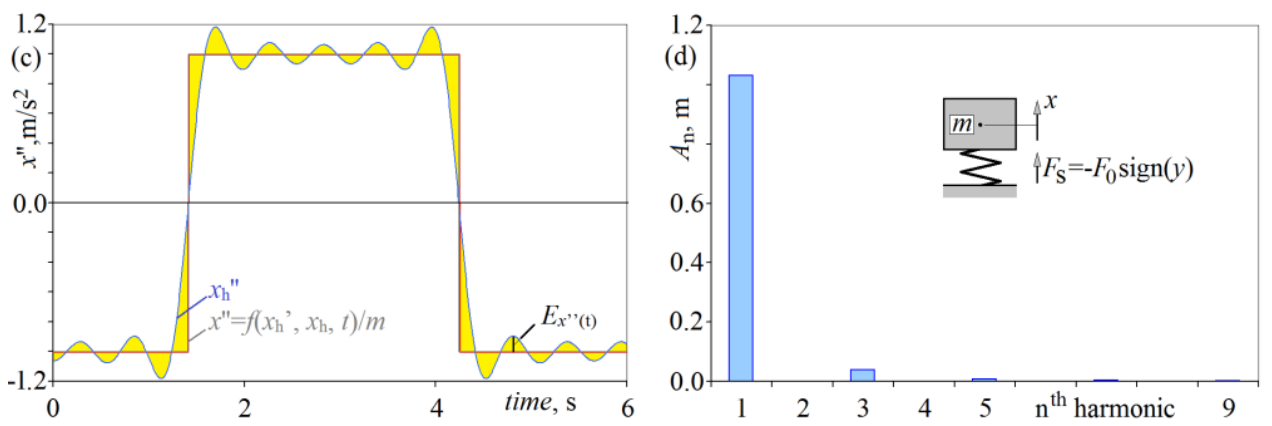

Fig. 3. Time histories of displacement (a), velocity (b), acceleration (c), and spectrum (d) obtained with HBM for the following conditions $m=1 \mathrm{~kg}, F_{0}=1 \mathrm{~N}, x_{(\mathrm{t}=0)}=1 \mathrm{~m}$ and $x^{\prime}(\mathrm{t}=0)=0 \mathrm{~m} / \mathrm{s}$, Eq. (8).
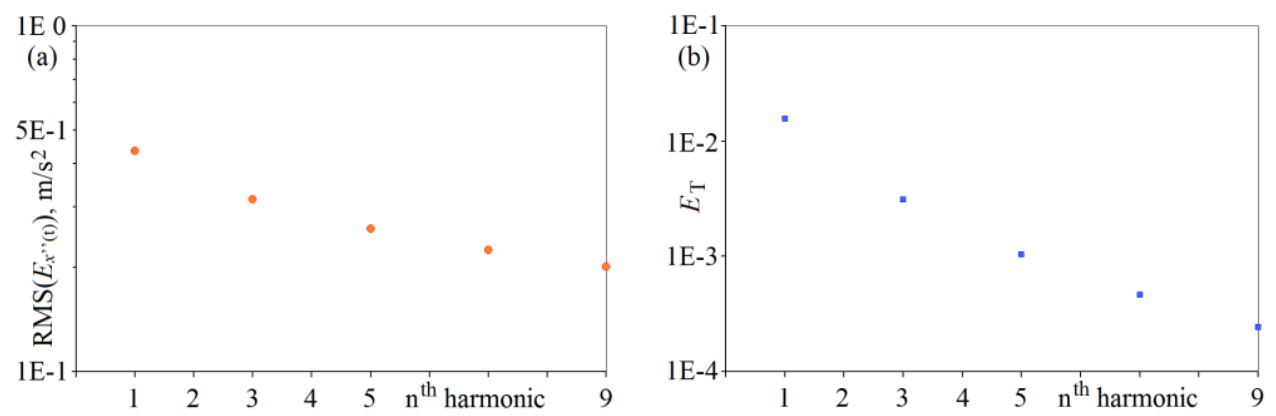

Fig. 4. $\operatorname{RMS}\left(E_{x^{\prime},(\mathrm{t})}\right)(\mathrm{a})$ and error of period $E_{\mathrm{T}}(\mathrm{b})$ against number of harmonic.

\section{Application of HBM}

Next, the periodic solution of the following equation is studied:

$$
x^{\prime \prime}=\left(-k_{3} x^{3}-c x^{\prime}+F_{\mathrm{E}} \sin (\omega t)\right) / m,
$$

where $c$ - denotes damming coefficient $\mathrm{N} /(\mathrm{m} / \mathrm{s})$, and $F_{\mathrm{E}}$-amplitude of excitation $\mathrm{N}$. For $k_{3}=1 \mathrm{~N} / \mathrm{m}^{3}, \mathrm{c}=0.05 \mathrm{~N} /(\mathrm{m} / \mathrm{s}), F_{\mathrm{E}}=7.5 \mathrm{~N}, \omega=1 \mathrm{rad} / \mathrm{s}$ and $m=1 \mathrm{~kg}$ chaotic vibrations are observed, thus HBM can be used to find unstable periodic solutions, which is within any chaotic attractor. Agreement between numerical and HBM solution is excellent for eleven harmonics which shows convergence (Fig. 5). Time histories are smooth but theirs shape is complex, thus eleven harmonics were used. Moreover, amplitudes of higher harmonics are significant. The periodical solution is unstable, because the numerical solution escapes to the chaotic attractor (Fig. 6). This effect is known as the butterfly effect. After a few cycles, a numerical nose is amplified, and the phase point leaves the periodic cycle. Obtained cycle is similar to homoclinic orbit, because of shape of $x$-x', trajectory (Figs.6d, 7a).
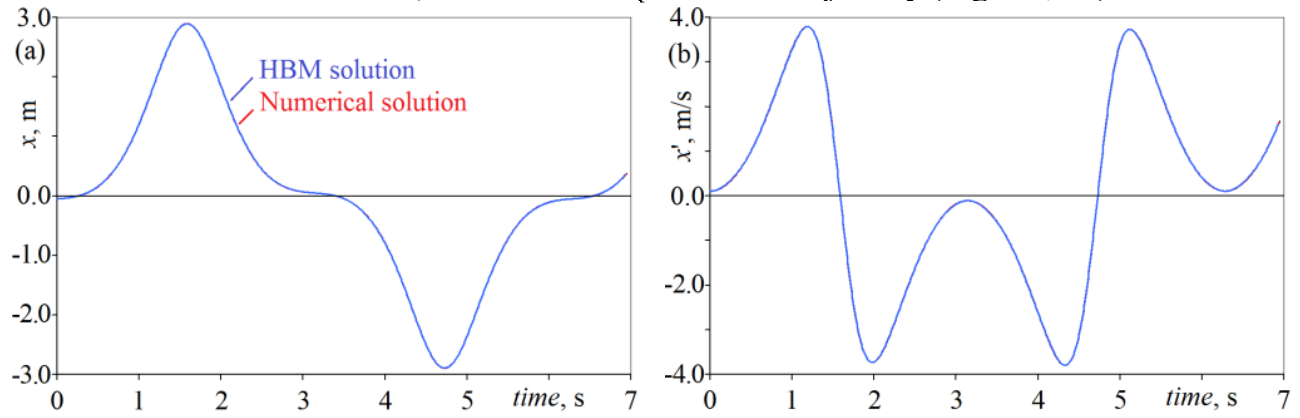

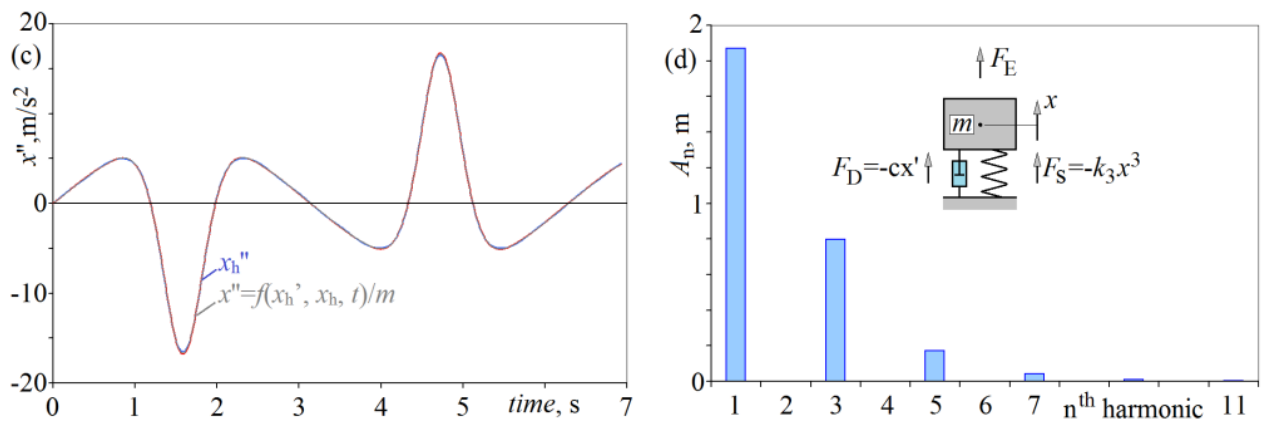

Fig. 5. Time histories of displacement (a), velocity (b), acceleration (c), and spectrum (d) obtained with the HBM and a direct method for $k_{3}=1 \mathrm{~N} / \mathrm{m}^{3}, \mathrm{c}=0.05 \mathrm{~N} /(\mathrm{m} / \mathrm{s}), F_{\mathrm{E}}=7.5 \mathrm{~N}, \omega=1 \mathrm{rad} / \mathrm{s}, m=1 \mathrm{~kg}$, Eq. (9).
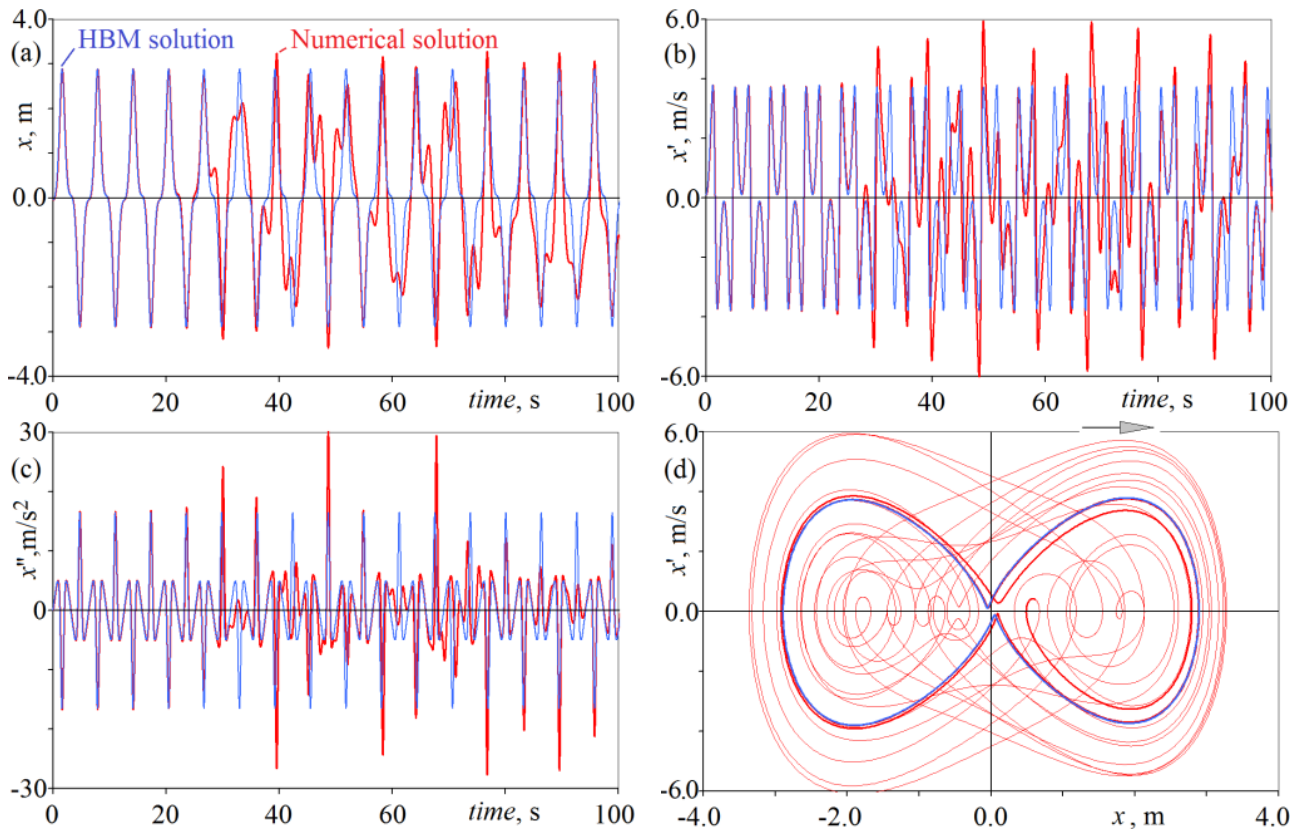

Fig. 6. Time histories of displacement (a), velocity (b), acceleration (c), and phase portrait (d) obtained with the HBM and a direct method for $k_{3}=1 \mathrm{~N} / \mathrm{m}^{3}, \mathrm{c}=0.05 \mathrm{~N} /(\mathrm{m} / \mathrm{s}), F_{\mathrm{E}}=7.5 \mathrm{~N}, \omega=1 \mathrm{rad} / \mathrm{s}$, $m=1 \mathrm{~kg}$, Eq. (9).

These vibrations can be studied with $x-x$ ' and $x^{\prime}-x$ ' trajectories. Trajectory $x-x$ ' of periodic solution is similar to spring characteristic with hysteresis (Fig.7a), whereas shape of chaotic attractor is more complex. For linear vibrations, this trajectory is a straight line. Whereas, trajectory $x^{\prime}-x^{\prime}$ ' shows complexity of this motion, inner loops are observed. For linear vibrations phase portrait $x^{\prime}-x^{\prime}$ ' has an elliptical shape. Summarising, shape of chaotic attractor correspond to shape of unstable periodic orbit. 

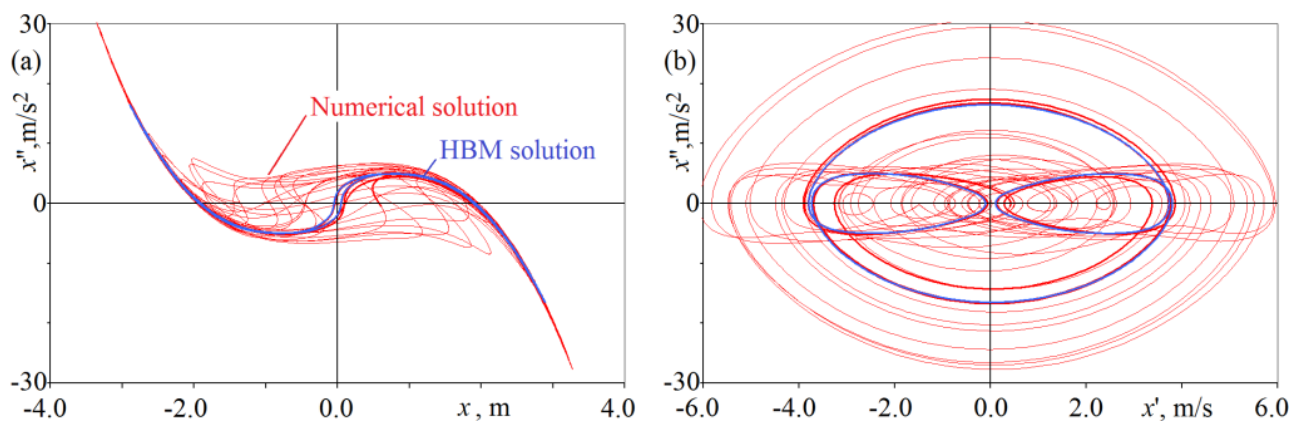

Fig. 7. Trajectories $x-x^{\prime \prime}$ (a) and $x^{\prime}-x^{\prime \prime}$ (b), obtained with HBM and a direct method for $k_{3}=1 \mathrm{~N} / \mathrm{m}^{3}$, $\mathrm{c}=0.05 \mathrm{~N} /(\mathrm{m} / \mathrm{s}), F_{\mathrm{E}}=7.5 \mathrm{~N}, \omega=1 \mathrm{rad} / \mathrm{s}, m=1 \mathrm{~kg}$, Eq. (9).

\section{Conclusions}

The HBM provides an opportunity to find stable and unstable periodic cycles, which play a significant role in the bifurcation theory. Unstable solutions are difficult to obtain with numerical methods, because the numerical solution escapes to an attractor. Obtained results can be compared with a perturbation solution, because perturbation solutions are sums of harmonics. Unfortunately, the HBM is ineffective for discontinues time histories. Summarising, unstable cycles and other phenomena are not observed in linear systems, thus non-linear models should be used in practice, because they better describe real vibrations than linear models.

\section{References}

1. R. Kostek, Appl. Math. Comput 219, 19 (2013).

2. K.J. Bathe, E.L. Wilson, Earthq Eng Struct Dyn, 1 (1972).

3. S.R. Panigrahi, B.F. Feeny, A.R. Diaz, J. Vib. Acoust, 137, 6 (2015). 